\title{
Total Unilateral Lung Atelectasis in a Child with Asthma and Its Rapid Resolution with Medical Management without Any Invasive Intervention: A Case Report
}

\author{
Sarmad Farook Yahya AlHamdani ${ }^{a}$ Rashid Nadeem ${ }^{b}$ \\ aDepartment of Pediatrics, Dubai Hospital, Dubai Health Authority, Dubai, UAE; \\ ${ }^{b}$ Department of Intensive Care Medicine, Dubai Hospital, Dubai Health Authority, \\ Dubai, UAE
}

\section{Keywords}

Unilateral lung atelectasis - Asthma - Conservative management

\begin{abstract}
Asthma is the most common chronic lung condition of childhood affecting approximately 6 million children in the United States. Right middle lobe syndrome or atelectasis is common in children with asthma though whole unilateral lung collapse is rare; only case series are reported. Bronchoscopy is usually required in addition to medical management of asthma and treatment of infection. We present a case of unilateral lung atelectasis in a young child which resolved with intense medical management without any endoscopy.

(C) 2019 The Author(s)

Published by S. Karger AG, Basel
\end{abstract}

\section{Introduction}

Asthma is the most common chronic lung condition of childhood, affecting approximately 6 million children in the United States [1]. Right middle lobe syndrome or atelectasis is 
common in children with asthma [2] though whole unilateral lung collapse is rare; only case series are reported [3-5]. Management usually include bronchoscopy in addition to medical management of asthma and its reason for exacerbation. We present a case of whole unilateral lung collapse in a young child which resulted in dramatic resolution without any endoscopy.

\section{Case Report}

A 9-year-old boy with a history of asthma for many years presented to another facility with cough and wheezing for the last 3 days. One day prior, he was playing with tissue paper and swallowed a small piece without obvious chocking. His mother had given him Ventolin and Atrovent nebulization at home without any improvement; therefore, she brought him to the emergency department. The patient was transferred to our facility for further management. Our evaluation revealed tachycardia (heart rate 122 beats/min), tachypnea (respiratory rate of $40 / \mathrm{min}$ ), and blood pressure $102 / 69 \mathrm{~mm} \mathrm{Hg}$ without any fever or cyanosis. Pulse oximetry was showing $94 \%$ on $100 \%$ oxygen via a nonrebreathing mask. Chest examination was remarkable for decreased breath sounds on the right side, and chest X ray revealed collapse of the right lung with shifting of the heart and mediastinum to the same side (Fig. 1). The patient was admitted to the high-dependency unit for possible bronchoscopy and was started on Ventolin nebulization, intravenous Cefuroxime and normal saline. Laboratory tests were normal except WBC $17,300 / \mathrm{mm}^{3}$, with Neutrophils $83 \%$. Mycoplasma IgM was positive. Arterial blood gas analysis on nonrebreather $100 \%$ oxygen showed: $\mathrm{pH}=7.36, \mathrm{PCO}_{2}=39.9 \mathrm{~mm}$ $\mathrm{Hg}, \mathrm{PO}_{2}=47.1 \mathrm{~mm} \mathrm{Hg}$. The differential diagnosis included severe acute exacerbation of asthma with right lunge collapse with possible foreign body aspiration or right-sided pneumonia with atelectasis. Management included nebulized bronchodilators, saline and antibiotics while bronchoscopy was also considered. After multiple serial exams (few minutes apart) by a pediatrician, an intensivist and an ear nose throat specialist, a multidisciplinary meeting decided to proceed with close clinical follow up of the patient with medical treatment while a bronchoscopy team stayed in the ICU as standby, as the patient was showing clinical signs of improvement (improving tachypnea and tachycardia) and the risk of the bronchoscopy procedure appeared to be greater than the benefits. The likelihood of a solid foreign body was low because of a reliable history given by the patient. Intense medical treatment included dexamethasone i.v. $10 \mathrm{mg}$, epinephrine $0.3 \mathrm{mg}$ subcutaneously given twice, $15 \mathrm{~min}$ apart, magnesium sulfate i.v. $50 \mathrm{mg} / \mathrm{kg}$, Atrovent $250 \mu \mathrm{g}$ with $5 \mathrm{mg}$ of Ventolin (three nebulizations, one after another) with chest physiotherapy. The patient dramatically improved in the following $3 \mathrm{~h}$ with associated improvement in oxygen requirement to only $2 \mathrm{~L} / \mathrm{m}$. Repeated X-ray of the chest showed complete resolution of right lung atelectasis (Fig. 2). Subsequently, the patient was followed up on the pediatric ward and then discharged home on asthma maintenance medication with follow-up appointment as an outpatient.

\section{Discussion/Conclusion}

Our case of asthma with unilateral complete right lung atelectasis is a rare presentation. The etiology of atelectasis in children with asthma includes risk factors of smaller and collapsible airways, more pliant chest walls, and inefficient collateral ventilation. Moreover, bronchial inflammation produces cellular debris, mucus plugs, and edema, which promotes the development of atelectasis in asthma [6]. Potential prognostic factors for the development of 
atelectasis in children with asthma are age, gender, age at diagnosis, family history for atopic diseases, smoke exposure, allergic rhinitis, asthma control parameters, level of asthma control, atopy, and serum immunoglobulin E levels. Similar factors and size or thickness of atelectasis are prognostic factors for the resolution of atelectasis [7]. The baseline profile of children with asthma who are at risk for significant atelectasis is unknown although two distinctive phenotypes predominate: one with eosinophilic inflammation associated with a gradual onset and a slow response to therapy and a second phenotype with neutrophilic inflammation that has a rapid onset and rapid response to therapy [8]. Our patient possibly has infection as suggested by an increase in leukocytosis with neutrophilia, although cultures from respiratory secretions and blood were negative. Studies suggest that about $50 \%$ of asthmatics who develop atelectasis have a coexistent infection, although evidence is frequently lacking because of early use of antibiotics. Infection promotes more inflammation, edema and production of secretion leading to atelectasis [9].

The management of lung atelectasis usually requires rigid or flexible interventional bronchoscopy [3-5] as foreign body is frequently involved. Bronchoscopy is a high-risk procedure in this situation but is required to prevent rapid decline in pulmonary status and its associated adverse consequences. Our case was unique in the sense that we have a reliable history including no solid foreign body. Our bronchoscopy team was readily available and agreed to stand by in case of emergency endoscopy. The patient had started to show clinical improvement; therefore, watchful waiting was less risky than bronchoscopy itself. The approach of careful observation and intense medical management including continuous bronchodilation, anti-inflammatory medications [7] and specialized chest physiotherapy [10] resulted in improvement within an hour, which was reassuring to continue with the noninvasive approach (Fig. 3). However, we advise extreme caution. Every clinician should assess the readiness of the equipment and the interventional bronchologist for procedure if the patient does not improve with medical treatment. The clinician should carefully assess each clinical case history, available data and change in clinical status with treatment on an hourly basis while keeping the bronchoscopy team on standby. With this strategy, our patient rapidly improved and invasive intervention was not required.

\section{Statement of Ethics}

A written informed consent was obtained from the family. There are no patient identifiers in the case report which may link the patient to the report.

\section{Disclosure Statement}

All authors have no conflict of interest and no financial interest in the subject matter to disclose.

\section{Funding Sources}

No funding was received for this study. 


\section{Author Contributions}

Dr. Sarmad Farook Yahya AlHamdani: Took care of the patient, conceived the idea, wrote the case report, extracted data, and analyses data and wrote and approved the manuscript.

Dr. Rashid Nadeem: conceived the idea, wrote the case report, extracted data, and analyses data and wrote and approved the manuscript.

\section{References}

1 Zahran HS, Bailey CM, Damon SA, Garbe PL, Breysse PN. Vital signs: asthma in children-United States, 2001-2016. MMWR Morb Mortal Wkly Rep. 2018 Feb;67(5):149-55.

2 Sekerel BE, Nakipoglu F. Middle lobe syndrome in children with asthma: review of 56 cases. J Asthma. 2004 Jun;41(4):411-7.

3 Aronsohn RB, Pressman JJ. Massive atelectasis in bronchial asthma. Ann Otol Rhinol Laryngol. 1958 Dec;67(4):1106-12.

4 Rakower J, Wayl P, Halberstadt H. Massive asthmatic atelectasis. Dis Chest. 1955 May;27(5):573-8.

5 Garofalo P, Ballario R. [The massive atelectasis syndrome due to "mucoid plug" in the course of bronchial asthma. (A case observed at the Clinica Pediatrica of Turin in an 11-year-old girl)]. Minerva Pediatr. 1971 Dec;23(51):2138-45.

6 Peroni DG, Boner AL. Atelectasis: mechanisms, diagnosis and management. Paediatr Respir Rev. 2000 Sep;1(3):274-8.

7 Soyer 0, Ozen C, Cavkaytar 0, Senyücel C, Dallar Y. Right middle lobe atelectasis in children with asthma and prognostic factors. Allergol Int. 2016 Jul;65(3):253-8.

8 Restrepo RD, Peters J. Near-fatal asthma: recognition and management. Curr Opin Pulm Med. 2008 Jan;14(1):13-23.

9 Springer C, Avital A, Noviski N, Maayan C, Ariel I, Mogel P, et al. Role of infection in the middle lobe syndrome in asthma. Arch Dis Child. 1992 May;67(5):592-4.

10 Oberwaldner B. Physiotherapy for airway clearance in paediatrics. Eur Respir J. 2000 Jan;15(1):196-204. 


\section{Case Reports in Acute Medicine}

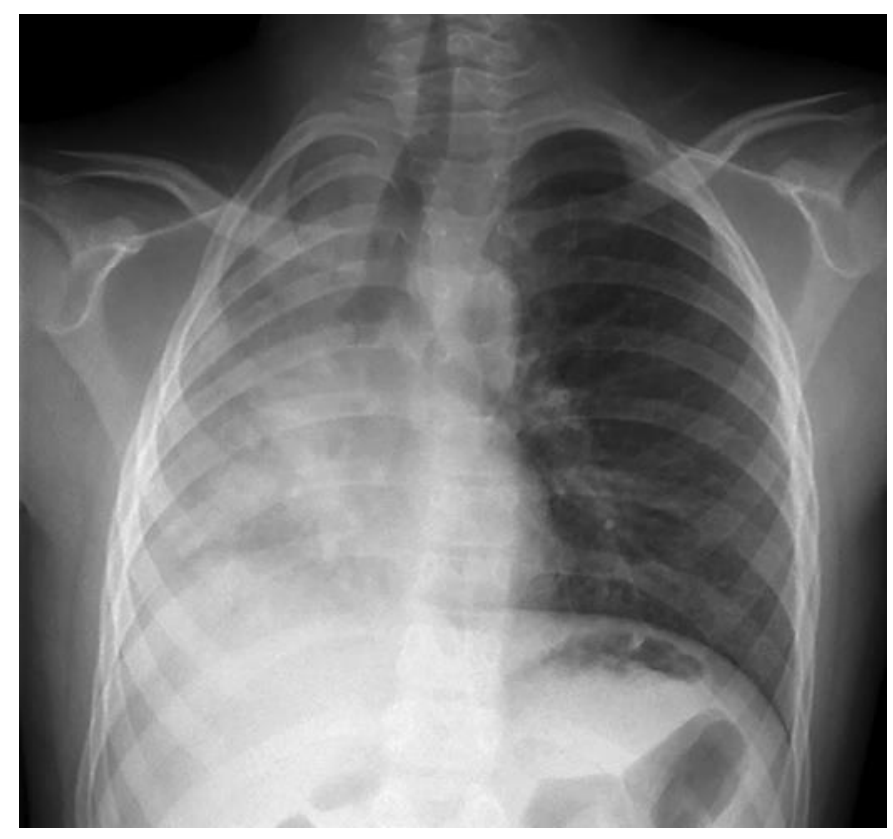

Fig. 1. X-ray of the chest: identify right-sided total lung atelectasis.

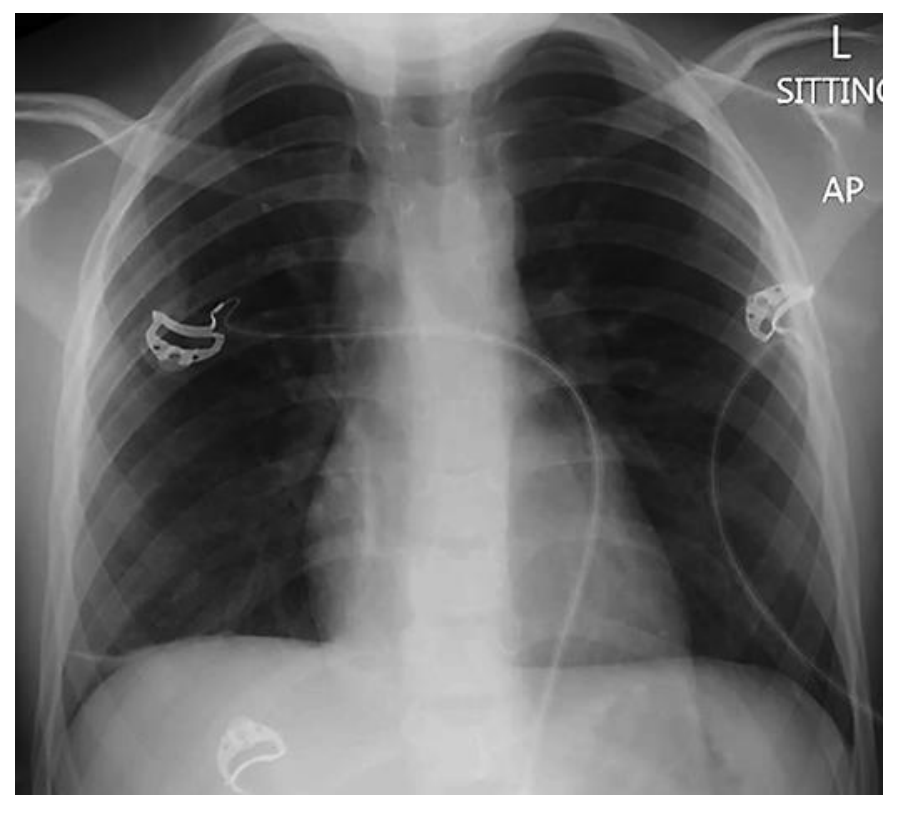

Fig. 2. X-ray of the chest: after $5 \mathrm{~h}$ of documenting resolution of right-sided atelectasis.

AlHamdani and Nadeem: Total Unilateral Lung Atelectasis in a Child with Asthma and Its Rapid Resolution with Medical Management without Any Invasive Intervention 


\section{Acrtemedicine}

AlHamdani and Nadeem: Total Unilateral Lung Atelectasis in a Child with Asthma and Its Rapid Resolution with Medical Management without Any Invasive Intervention

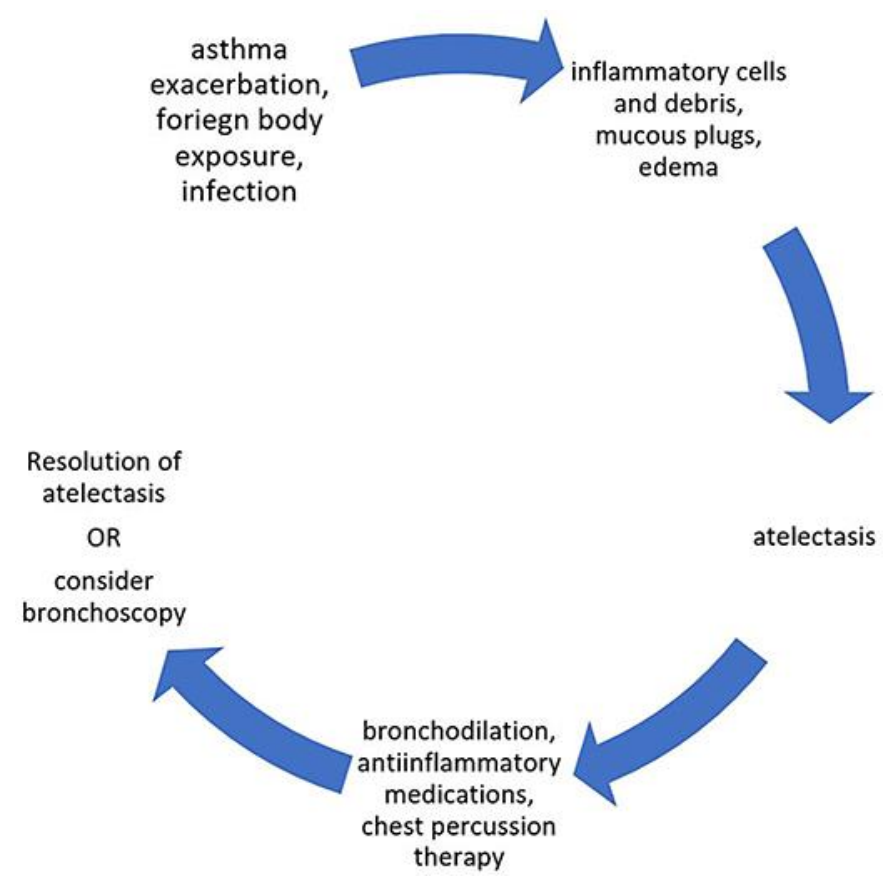

Fig. 3. Clinical course of atelectasis development and resolution. 